\title{
Etude Exploratoire De L'impact Des Systèmes d'Information Sur La Performance À Partir D'une Approche Par Alignement : Cas Des Entreprises Marocaines
}

\author{
Yassine Regragui, PhD Student \\ Youssef Al Meriouh, PhD Professor
}

Ecole Nationale de Commerce et de Gestion de Tanger

Université Abdelmalek Essaadi, Maroc

Doi: 10.19044/esj.2017.v13n31p261 URL:http://dx.doi.org/10.19044/esj.2017.v13n31p261

\begin{abstract}
The issue of the assessment of the contribution of the information systems to performance (IS) is an area of investigation which has been explored a little in Morocco. From an academic point of view, it is a matter of going further into the field of investigation by the enrichment of previous studies on the issue of the alignment of IS and the performance of organizations. From a practical point of view, it meets the expectations of businesses by providing answers to the question of contribution of IS in obtaining high-performance results. Based on the model of strategic alignment, a qualitative study of the exploratory character of hybrid type was conducted with thirteen Moroccan companies of different sizes and operating managers in different sectors of activities. This is in a bid to assess the applicability of research including the relevance and inadequacies of constructs in the field. The results of this study allowed us to adapt our research hypotheses to the Moroccan context. As a result, a number of variables have been integrated. These makes it possible to build our final alignment of IS model and phase confirmatory.
\end{abstract}

Keywords: Performance, information systems, alignment, contingency theory, stakeholder theory

\section{Résumé}

La question de l'évaluation de l'apport des systèmes d'information (SI) à la performance constitue un domaine d'investigation encore peu exploré au Maroc. D'un point de vue académique, il s'agit d'aller plus loin dans le champ d'investigation par l'enrichissement des études 
antérieurs portant sur la problématique de l'alignement des SI et la performance des organisations. D'un point de vue pratique, il s'agit de répondre aux attentes des entreprises en apportant des éléments de réponses à la question de contribution des SI à l'obtention des résultats performants. Tout en se basant sur le modèle de l'alignement stratégique, une étude qualitative à caractère exploratoire de type hybride a été menée auprès des responsables de treize entreprises marocaines de tailles différentes et opérantes dans des secteurs d'activités différents, afin d'apprécier l'applicabilité de la recherche notamment la pertinence et les insuffisances des construits sur le terrain. Les résultats de cette étude nous ont permis d'adapter nos hypothèses de recherche au contexte marocain. Ainsi un certain nombre de variables ont été intégrées. Celles-ci permettent alors de construire notre modèle final d'alignement des SI et d'entamer la phase confirmatoire.

Mots-clés: Performance, systèmes d'information, alignement, théorie de la contingence, théorie des parties prenantes

\section{Introduction}

Dans un environnement caractérisé par la mondialisation et l'émergence d'une économie dite de l'information, toute entreprise a besoin d'un système d'information (SI) capable de permettre un soutien complet et efficace dans la gestion opérationnelle et stratégique afin de soutenir l'adaptation de l'organisation aux exigences du contexte actuel. Pour cela, les entreprises ne cessent d'investir dans les SI pour améliorer leur performance qui est devenue plus une nécessité qu'un objectif à atteindre, notamment dans un contexte où la seule certitude est l'incertitude, les SI sont censées fournir un avantage stratégique pour les entreprises en tant que levier de performance et de transformation des processus organisationnels (Baradjay, 2000; Wang et al., 2008; Santhanam \& Hartano, 2003; Muhanna \& Stoel, 2010). A cet effet, il est important pour tout chef d'entreprise d'appréhender de manière rigoureuse ce mécanisme d'alignement des SI et son effet sur la performance.

A cette effet, une enquête a été réalisée par l'association des utilisateurs des SI au Maroc en 2012, a constaté que l'entreprise marocaine dédit $0,86 \%$ de son chiffre d'affaires au budget SI. Ce ratio varie entre $0,01 \%$ et $2,1 \%$ selon les secteurs d'activité dont les grandes entreprises consacrent en moyenne $1,04 \%$ de leur CA à la dépense SI. Concernant les secteurs champions de l'investissement SI au Maroc sont les télécommunications, les institutions financières et les administrations publiques. La destination de ces investissements porte principalement en 
matériel informatique \& infrastructure qui continuent de s'accaparer la part du lion dans le budget SI de la majorité des entreprises marocaines (47\%).

En effet l'évaluation de l'effet des SI sur la performance constitue un domaine d'investigation encore peu exploré au Maroc. Ainsi dans le but de répondre à un double objectif. D'un point de vue académique, il s'agit d'aller plus loin dans le champ d'investigation par l'enrichissement des travaux antérieurs traitant la problématique de l'alignement des SI et la performance des entreprises. D'un point de vue pratique, il s'agit de répondre aux attentes des entreprises en apportant des éléments de réponses à la question de contribution des SI à l'obtention des résultats performants?

Ainsi, plusieurs travaux de recherches ont traité la question de l'apport des investissements en SI sur la performance des entreprises. Les meilleures conclusions conduisent que ce n'est pas l'importance des investissements en SI eux-mêmes qui ont un véritable effet sur la performance, mais plutôt leur adéquation avec la stratégie de l'entreprise, (Verreault \& Rivard, 2003).

Dans ce sens, certains chercheurs comme le cas de (Iivari, 1992; Henderson \& Venkatraman, 1993) se sont interrogés sur les approches basées sur la théorie de la contingence en management stratégique. Le but est de comprendre la contribution des SI à la performance via ce mécanisme d'alignement entre les SI et d'autres variables comme la stratégie, la structure de l'entreprise ou encore son environnement.

S'inscrivant dans cette logique, ce travail de recherche est basé sur le concept de l'alignement en tant qu'une nouvelle manière d'étudier la performance induite par les SI et tente à répondre à la question centrale suivante: Dans quelle mesure l'alignement des SI contribue à l'amélioration de la performance des entreprises dans le contexte marocain?

\section{Cadre théorique de la Recherche}

Dans le but de répondre à notre problématique nous avons opté à un positionnement théorique de causes à effets, (de Vaujany, 2009). Celles-ci tentent de montrer un lien statistique significatif entre le SI et sa contribution, c'est-à-dire la performance qu'il engendre, dont le modèle d'alignement stratégique qui fait partie de ce courant. Et en d'autre part l'approche sociotechnique qui fait partie des approches processuelles a été aussi mobilisée qui prend en compte l'effet de chaque sous-système. Cela implique que la performance de l'organisation associée à l'intégration d'une technologie résulte de l'interaction non déterministe des deux sous-systèmes à savoir technique et social ainsi, elle s'inscrit dans une vision systémique de l'organisation. 
Pour cette étude nous avons pris le parti de proposer notre modèle de recherche dans une approche sociotechnique de l'alignement stratégique qui fait croisement de deux perspectives théorique de manière simultané (Renaud, 2012), le modèle d'alignement stratégique basé sur la théorie de la contingence et le recours à la théorie des parties prenantes en tant que théorie complémentaire de notre cadre théorique.

En effet, la contingence est une perspective complètement adéquate pour l'explication de la performance (Weill \& Olson, 1989). En ce sens, Chandler, a déjà annoncé en 1962 que l'harmonisation des choix de positionnement externe et interne ont contribué à la réalisation de résultats performants pour les firmes. Ainsi, de nombreuses études en SI ont choisi d'adopter la perspective de la contingence dans l'analyse de la relation entre l'alignement et la performance.

Selon (Fimbel, 2007) « l'analyse des relations et interactions entre les parties prenantes, impliquées dans une manœuvre d'alignementsynchronisation du SI, doit dépasser les seuls cadres formels pour prendre en considération d'autres dimensions telles que les dimensions cognitives, psychologiques, sociologiques ainsi que culturelles» ce qui nous pousse à revenir à nouveau à la définition du SI, proposée par (Reix \& Rowe, 2002): « un SI est un ensemble d'acteurs sociaux qui mémorisent et transforment des représentations via des technologies de l'information et des modes opératoires » ce qui donne plus d'importance à aspect humain du SI.

Dans ce sens (Mercier, 2005), intègre une dimension éthique qui semble indispensable pour l'étude de la dynamique d'interaction entre les parties prenantes responsables de l'alignement stratégique du SI qui s'inscrit, selon (Fimbel, 2007), dans une perspective réaliste d'un champ de forces ouvert dans lequel les interactions et rétroactions sont nombreuses et diverses.

En se basant sur les travaux issus de Cigref 2003, pour mieux justifier cette réflexion à travers le recours à la théorie des parties prenantes. Selon Cigref, une partie prenante est définie comme : " personne ayant un intérêt dans le fonctionnement du système d'information, personne pour qui le système d'information est un enjeu ». C'est ainsi qu'il a construit sa démarche autour de trois étapes à savoir l' identification de l'ensemble des parties prenantes du SI, la définition des rôles et responsabilités de chacune d'elles et enfin l'organisation des relations entre elles autour de processus et au sein de structures, le but c'est la réduction des risques liés aux projets et, notamment, accroître la valeur du SI (Cigref, 2003).

A signaler que Fimbel (2007), a mis l'accent sur le concept d' " impact » pour faire la distinction entre le concept de partie prenante, du concept d'acteur. En fait, ce chercheur a mis en évidence une distinction 
intéressante entre la notion d'acteur et celle de partie prenante comme en témoigne, entre autres, du centrage de son modèle "DyGAM" sur l'approche par les parties prenantes. Fimbel déclare que la notion d'acteur est relative à celui qui fait, qui intervient, alors que selon (Freeman, 1999), le pionnier de la version contemporaine de la Stakeholders Theory, "Une partie prenante est un individu ou groupe d'individus qui peut affecter ou être affecté par la réalisation des objectifs organisationnels». Ainsi ce même auteur stipule que ce modèle d'alignement stratégique étudie des relations entre des domaines et non entre des parties prenantes impliquées collectivement dans le management du SI.

\section{Les SI sous les approches :Alignement stratégique et socitechnique.}

L'alignement stratégique est toujours une question d'actualité et présente une importance capitale pour le management stratégique des SI (Bergeron et al., 2004; Teo \& King, 1997; Reich \& Benbasat,1996). Il constitue une base solide du paradigme stratégique actuel (Raymond et al., 2010) et une condition indispensable pour la croissance des entreprises (Sabherwal \& Chan, 2001).

En effet, l'évolution du rôle stratégique des SI a encouragé les dirigeants d'entreprises à s'intéresser de plus en plus de la relation entre l'entreprise et les SI en matière de coordination et de planification. Ainsi, l'alignement stratégique propose l'un des fondements théoriques les plus importants de la compréhension du rôle stratégique des SI et de son impact sur la performance des firmes (Raymond et al., 2010). Toutefois, il y a une constatation de l'absence d'une définition précise et communément acceptée dans la littérature portant sur le concept de l'alignement stratégique. Ce qui laisse, ce dernier défini de façons différentes dans la littérature (Chan \& Reich, 2007).

En s'appuyant sur les principaux travaux dans ce domaine à savoir (Henderson \& Venkatraman, 1993) qui ont proposé un modèle qui estime que le développement des SI et de la stratégie de l'entreprise doit être en harmonie. Dans le cas contraire, cela crée des difficultés pour les entreprises, à tirer profit de leur SI en tant que levier de compétitivité et de performance. Pour cela ont identifié de manière plus rigoureuse les composantes de l'alignement à savoir la stratégie de l'entreprise, la stratégie SI, la structure organisationnelle et la structure du SI. Pour réaliser l'alignement stratégique, ça se passe par la recherche d'une cohérence (relation en double sens) entre quatre domaines principales à savoir:

La stratégie de l'entreprise qui comprend la position de l'entreprise sur le marché en termes le couple produit/marché, ses compétences spécifiques et son métier; 
La structure organisationnelle correspond à la structure administrative, la définition des processus, etc.;

La stratégie des systèmes d'information se rapporte aux choix des technologies accessibles et au mécanisme de gouvernance;

L'architecture du système d'informations, liée au portefeuille d'applications, et l'architecture technologique.

Une des nouveautés apporté par ce modèle c'est la séparation qu'il propose dans la fonction SI avec d'un coté la stratégie et la gouvernance et de l'autre côté, tout ce qui est liée à l'infrastructure et processus.

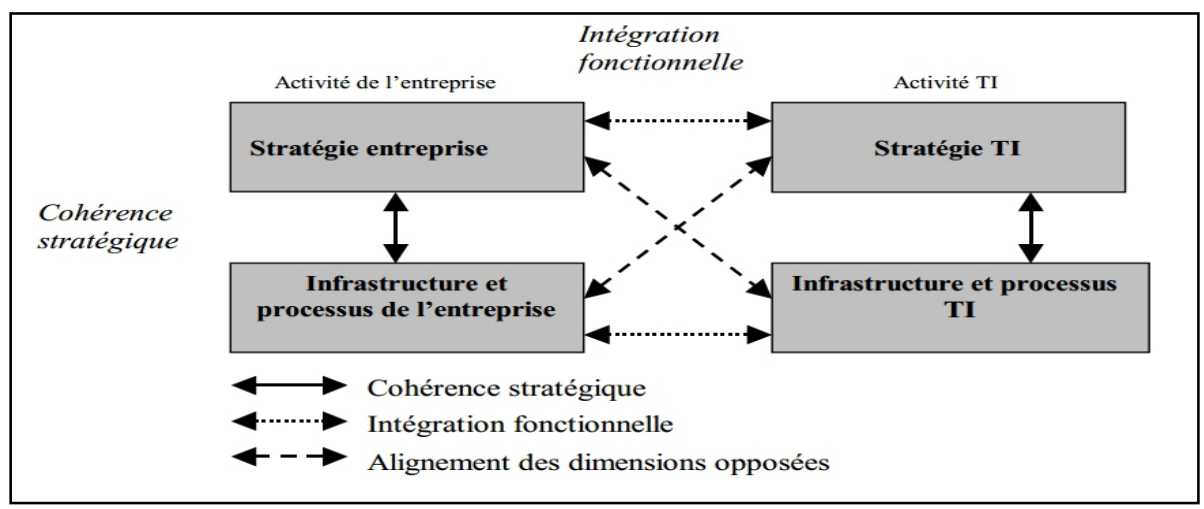

Figure 1. Modèle d'alignement stratégique (Henderson \& Venkatraman, 1993)

Par ailleurs, à noter que l'alignement stratégique est composé de deux voies autour des quatre dimensions que nous avons mentionnées:

La cohérence stratégique entre les dimensions de l'entreprise (stratégie) et les dimensions internes (déploiement de la technologie);

$\square \quad$ L'intégration fonctionnelle des SI au sein de l'entreprise, assurer et maintenir l'harmonie entre la structure organisationnels et l'architecture des SI.

La vision de ce modèle à travers ces différentes relations n'est pas de découvrir un niveau d'alignement parfait, le «one best way », mais de trouver un alignement propre qui correspond à chaque entreprise, et il est donc nécessaire de combiner des liens stratégiques, structurels et technologiques.

Ce modèle d'alignement stratégique était utilisé pour la base de plusieurs études empiriques qui ont tenté de mesurer l'apport de l'alignement entre les SI (structure ou stratégie) et stratégie d'entreprise ou l'infrastructure organisationnelle sur la performance (Jouirou \& Kalika, 2004).

A cet effet, (Henderson \& Venkatraman, 1993), ont pris appui sur ce modèle pour plusieurs de leurs travaux de recherches et ont tenté d'expliquer comment l'alignement des SI avec les choix stratégiques et la structure organisationnelle peut contribuer à une meilleure performance 
organisationnelle. En ce sens (Raymond, 2002), a souligné la nécessité d'analyser l'effet sur la performance passant par un examen de la cohérence entre les différentes dimensions, telles que les décisions stratégiques, structurelles et technologiques. Quelle que soit la méthode ou la perspective recommandée pour traiter ce fit (médiateur, modérateur, co-variaton, etc.). L'alignement stratégique est une approche qui a le mérite de mettre les SI au centre des préoccupations stratégiques des gestionnaires, il n'a pas reçu l'attention et l'apport des intervenants dans ce processus, du fait que ce dernier n'a pas abordé la question d'alignement de manière dynamique.

Dans le but de rester fidèle au modèle de l'alignement stratégique, les définitions des concepts du modèle correspondent à celles proposées par (Henderson \& Venkatraman, 1993). Toutefois il est nécessaire de faire des ajustements pour la construction de notre modèle de recherche dans le but de donner plus de précision et de clarté à son application dans le contexte marocain.

(Regragui, 2016) s'inscrit dans la même logique des travaux antérieurs comme le cas de (Leidner \& Kayworth, 2006) considère qu'une meilleure interaction entre les responsables manageriels et techniques devrait être modéré par l'effet de la culture individuelle des parties prenantes afin de réussir ce processus d'alignement stratégique d'où l'importance de l'intégration de la dimension culturelle dans le modèle de base ainsi que la prise en compte des variables de contrôle relatives au contexte de l'entreprise à savoir sa taille son secteur de l'activité à l'instar des études menées par (Kalika et al., 2002; Rival, Y. 2005, 2008).

L'approche sociotechnique a pour postulat que toute organisation est le fruit de l'addition de deux sous-systèmes : le système social et technique nécessite une interdépendance relativement grande entre tous les éléments du système social et technique. La technologie agit sur la structure sociale et inversement. Ce courant sociotechnique prend en compte l'effet de chaque sous-système, ainsi, il s'intègre dans une vision systémique de l'organisation.

Ici, nous allons définir les deux sous-systèmes mentionnés ci-dessus, le système technique et le système social.

- Le système technique est caractérisé par «processus, tâches et la technologie nécessaire pour la transformation des intrants en extrants ».

- Le système social est déterminé par les caractéristiques des personnes (compétences, attitudes, valeurs), les relations entre les acteurs et la structure.

Notre travail de recherche se base sur une approche d'alignement stratégique pour 1'explication de la performance induite par les SI en mobilsant l'approche sociotechnique comme une perspective alternative émergeant par une implication solide de l'acteur humain dans le processus 
d'alignement notamment en terme d'intercation humaine, sociale et intelectuelle des parties prenantes.

\section{La performance variable dépendante dans la recherche en systèmes d'information}

La performance constitue un thème récurrent de la recherche en sciences de gestion mais également un sujet de préoccupation pour les managers soumis à l'obligation de performance des structures qu'ils dirigent. On comprend dès lors que le sujet ait donné lieu à un grand volume de littérature, sans avoir pu pour autant arriver à un compromis. D'une manière générale, la performance a été souvent qualifiée d'un caractère multidimensionnel, qui est fréquemment utilisé pour désigner un ou plusieurs concepts spécifiques (Chan et al., 1997; Sabherwal et al., 2001), et selon diverses perspectives (Venkatraman \& Ramanujam, 1986).

En effet, il est clair que la notion de performance intègre par définition une certaine « subjectivité ». (Saulquin \& Schier, 2005) indiquent pour cela que «la performance a autant de facettes qu'il existe d'observateurs à l'intérieur et à l'extérieur de l'organisation. Elle est ainsi définie par ceux qui vont utiliser l'information.» Pour eux : «Le concept (de performance) possède ainsi autant de significations qu'il existe d'individus ou de groupes qui l'utilisent.

Pour un dirigeant, la performance pourra être la rentabilité ou la compétitivité de son entreprise ; pour un employé, elle pourra être le climat de travail ; et pour un client, la qualité des services rendus. La multiplicité des approches possibles en fait un concept surdéterminé, et curieusement, il demeure indéterminé en raison de la diversité des groupes qui composent l'organisation» Les travaux de (Bergeron et al., 2004) définissent la performance en termes d'efficience et d'efficacité et avancent que la performance est définie comme étant la force et la prospérité de l'entreprise à long terme face à ses concurrents. Elle regroupe deux dimensions à savoir : la croissance des ventes et la rentabilité. La première renvoie à la notion de prospérité alors que la seconde est relative à la force à long terme de l'entreprise. Aussi, à partir d'une revue de la littérature, on constate qu'il y a un consensus entre les chercheurs notamment sur les deux termes indispensables pour définir la performance à savoir : l'efficience et l'efficacité (Gervais, 2001).

D’après (Jouirou \& Kalika, 2004), la performance peut être mesurée par des indicateurs quantitatifs de nature objectifs qui se fondent sur des données financières et par des indicateurs qualitatifs d'ordre subjectifs qui font recours à l'appréciation de la perception des dirigeants. Pour mesurer ce concept, ces auteurs ont utilisé l'instrument de (Venkatraman,1989). En effet, cet instrument a permis d'évaluer la 
position compétitive et concurrentielle de l'entreprise et par conséquent, sa performance par rapport à l'évolution des ventes et de rentabilité.

Bennani et al. (2004) ont analysé plusieurs publications traitant la question de la contribution des SI à la performance, la totalité des études antérieures montre qu'il existe un impact positif et significatif de l'alignement des SI sur la performance. Ainsi a signaler que la majorité de ces travaux de recherches se limite à l'étude de deux facteurs notamment la stratégie de l'entreprise et la stratégie SI ou entre la structure de l'entreprise et la structure SI.

\begin{tabular}{|c|c|}
\hline Principaux Chercheurs & Résultats \\
\hline Karaoui, C. (2012) & \\
Rival, Y. (2005) (2008) & Chacun de ces chercheurs a suivi une \\
Jouirou, N. et Kalika, M. (2004) & démarche méthodologique différente \\
Kefi, H. et Kalika, M. (2003) & pour l'étude de la performance de \\
l'entreprise induite par les SI à travers \\
diverses perspectives d'alignement tout \\
en mobilisant notamment la théorie de \\
Bergeron, F., Raymond, L., et Rivard, S. (2002) \\
Croteau, A.M., Bergeron F., et Raymond, L. \\
(2001) contingence pour mieux comprendre \\
Sabherwal, R. et Chan, Y.E. (2001) & $\begin{array}{c}\text { la contribution des SI à la performance } \\
\text { Luftman, J. (2000) }\end{array}$ \\
des organisations. Dans ce sens la \\
majorité des travaux confirment \\
Teo, T. et King, W.R. (1996) & l'existence d'une relation étroite entre \\
Bergeron, F. et Raymond, I. (1995) & l'alignement et performance de \\
Papp, R. (1995) & l'entreprise et par conséquent un impact \\
positif et significatif sur la performance \\
de l'organisation.
\end{tabular}

Tableau 1. Principales conclusions des études portant sur l'alignement des SI et la performance

\section{Cadre empirique de la recherche}

Notre objectif à travers cette étude vise à mieux apprécier l'applicabilité de la recherche notamment la pertinence et les insuffisances des construits sur le terrain. A cet effet nous avons opté dans cette étude à une démarche qualitative à caractère exploratoire de type hybride à travers des allers-retours tout au long de la recherche dans le but de donner du sens aux observations empiriques tout en se basant sur des connaissances théoriques dans la littérature qui concerne l'objet de la recherche (Charreire \& Durieux, 2007).

\section{L'échantillonnage et le souci de la représentativité}

L'entretien semi-directif est un moyen de recherche qui apporte une grande richesse à l'analyse, à ce propos, la question se pose à ce dernier de savoir quel est le nombre minimum d'entretiens nécessaires en restant pertinent par rapport au sujet de recherche et à la méthode choisie (Allouani, 
2013). Cette étude qualitative exploratoire n'a pas pour objet de tester un modèle proposé à l'issue de la revue de littérature. Pour la taille de notre échantillon le choix des éléments est basé sur le jugement du chercheur par rapport à leur caractère typique ou atypique (échantillon par choix raisonné). Ainsi dans notre choix du nombre et de la qualité des personnes à interroger, nous avons tenu de respecter les deux critères recommandés pour toute recherche qualitative, à savoir le critère de la saturation et celui de la diversification.

Ainsi, dans le but d'avoir plus d'avis, la diversification des profils des répondants est introduit comme critère majeur de sélection en ce qui touche les échantillons qualitatifs d'où l'idée de diversifier les cas de manière à inclure la plus grande variété possible. Quant à la saturation, elle est décrite comme le "phénomène qui apparait au bout d'un certain temps dans la recherche qualitative lorsque les données que l'on recueille ne sont plus nouvelles ». Tous les efforts de collecte d'information nouvelle sont donc rendus inutiles. L'ensemble d'entretiens ont été conduit jusqu'à ce que les dernières interviews ne permettent plus d'intégrer de nouveaux apports théoriques dans le guide de l'interviewer (saturation théorique). Dans notre cas, nous avons atteint la saturation au bout du $12^{\text {ème }}$ entretien, nous nous en sommes rendu compte lors des entretiens $n^{\circ} 13$ et 14 .

\section{La collecte des données de l'étude exploratoire}

Le choix de la méthode des entretiens semi-directifs pour la collecte des données suppose la préparation préalable d'un guide d'entretien où le chercheur prépare les questions à aborder avec les interviewés (annexe 1). Les entretiens semi-directifs sont menés sur la base d'un guide d'entretien constitué de "différents thèmes questions » préalablement élaborées en fonction du cadre conceptuel et théorique préalablement posé (Albarello, 2004).

Ainsi, le choix des répondants et des entreprises objet de cette étude s'est effectué à partir des contacts professionnels existants. Ainsi, le nombre d'entreprises gardés nous paraît suffisant, puisque notre objectifs est d'apprécier l'application des variables de notre modèle théorique et de les confronter aux pratiques des entreprises sur le terrain et potentiellement l'intégration de nouvelles variables le cas échéant ou de stabiliser celles du modèle initial.

Ces entretiens ont été effectués de façon à ce que l'échantillon observé soit suffisamment diversifié. En effet, «la variété des entretiens est un élément important lorsque les entretiens sont utilisés pour générer les items sur lesquels le chercheur, dans la suite de sa recherche, recueillera des données par questionnaire» (Roussel \& Wacheux, 2006). Ainsi, les responsables métiers et SI interviewés travaillent dans des entreprises 
relativement différentes notamment en terme de taille ou de secteur d'activité (Tableau 1).

\begin{tabular}{|c|c|c|c|c|}
\hline & Poste occupé du répondant & Sexe & Expérience & Secteur d'activité \\
\hline E1 & Directeur Organisation et SI & $\mathrm{M}$ & 12 ans & Agroalimentaire \\
\hline E2 & Directeur des SI & $\mathrm{M}$ & 11 ans & Bancaire \\
\hline E3 & Directeur des Sites et des SI & M & 13 ans & Services \\
\hline E4 & $\begin{array}{l}\text { Chef Département études } \\
\text { et réalisation SI }\end{array}$ & M & 11 ans & Distribution \\
\hline E5 & Responsable Contrôle de gestion & M & 6 ans & Assurance \\
\hline E6 & Chef de projets SI & M & 9 ans & Agroalimentaire \\
\hline E7 & Directeur SI et communication & $\mathrm{M}$ & 12 ans & Bancaire \\
\hline E8 & $\begin{array}{l}\text { Responsable administratif et } \\
\text { financier }\end{array}$ & M & 8 ans & Pharmaceutique \\
\hline E9 & $\begin{array}{c}\text { Responsable Qualité et Sécurité } \\
\text { des SI }\end{array}$ & $\mathrm{F}$ & 7 ans & Assurance \\
\hline E10 & $\begin{array}{c}\text { Responsable ressources et } \\
\text { logistique }\end{array}$ & $\mathrm{F}$ & 6 ans & Transport \\
\hline E11 & Chef de projet SI & $\mathrm{F}$ & 9 ans & Telecom \\
\hline E12 & Chef département support & $\mathrm{F}$ & 14 ans & Distribution \\
\hline E13 & Chef de division SI & $\mathrm{F}$ & 12 ans & Transport \\
\hline
\end{tabular}

Tableau 2. Synthèse des caractéristiques de l'échantillon de l'étude exploratoire

Il convient de préciser qu'une réunion préliminaire a été tenu avec un consultant SI pour avoir une idée de manière générale sur les pratiques en matière des SI dans les entreprises et notamment la contribution des SI à la performance de l'entreprise à travers le mécanisme d'alignement et ce dans le cadre d'un entretien ouvert.

À la suite, Treize entretiens semi-directifs auprès des responsables métiers et SI ont été réalisés. Dix interviews ont eu lieu en face à face dont seulement quatre qui ont été enregistrés avec l'accord de l'interlocuteur puis ont été intégralement retranscrits, pour les six autres entretiens qui n'ont pas pu enregistrés nous avons opté dans ces cas pour la prise de note. Les entretiens ont été effectués entre Mars et Juillet 2016 avec une durée moyenne d'un peu prés d'une heure et se sont effectués dans des locaux différents selon la disponibilité du répondant. En outre nous avons réalisé le reste des entretiens à savoir trois par conversation téléphoniques.

\section{Méthode d'analyse de contenu}

Dans le cadre de l'analyse des données de type qualitatives la méthode la plus utilisée et connue est celle de l'Analyse de Contenu qui est la plus répandue pour l'étude des entretiens ou les observations qualitatives 
(Badrin, 2003). Elle consiste à retranscrire les données qualitatives, à se donner une grille d'analyse, à coder les informations recueillies et à les traiter.

L'analyse de la série des treize entretiens réalisés ont permis de réunir un ensemble d'informations que nous avons retranscrit et soumis à une analyse thématique qui a été retenu déjà comme type d'analyse de contenu et qui est la plus couramment utilisée dans les recherches en gestion (Blanchet \& Gotman, 1992).

Cette analyse peut être qualitative ou quantitative (Evrard, Pras \& Roux, 2003). Dans le premier cas, il s'agit de porter son attention sur l'importance des thèmes repérés dans les entretiens. Dans le second cas, l'objectif est d'évaluer la fréquence d'apparition de ces thèmes. Nous avons privilégié pour notre étude une analyse qualitative plutôt que quantitative pour l'analyse de contenu habituellement employé et mettant en avant l'importance des thèmes identifiés. Ainsi il convient de préciser que nous avons accordé plus d'attention à l'importance des thèmes repérés dans les entretiens plutôt qu'à la fréquence d'apparition de ces thèmes.

Un va-et-vient entre la codification des thèmes et la réalisation des entretiens s'est fait jusqu'à ce que les derniers entrevues n'apportent plus de nouvelle codification à fournir pour le dictionnaire des thématiques (principe de saturation). A cet effet nous avons utilisé pour cette fin, le logiciel Nvivo Qsr 10 qui est bien adapté à ce genre d'analyse par thématique (cf. Figure 2).

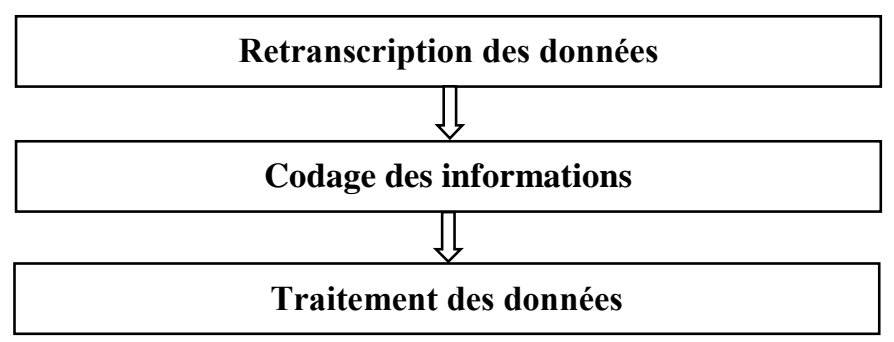

Figure 2. Etapes de l'Analyse des données qualitatives

\section{Discussion des résultats}

La structure générale de l'échantillon utilisé pour les fins de cette étude. Le tableau suivant relate les principaux résultats des statistiques descriptives effectuées en termes de pourcentage. 


\begin{tabular}{|c|c|c|c|}
\hline & Catégorie & Fréquence & $\%$ \\
\hline \multirow{6}{*}{$\begin{array}{c}\text { Secteur } \\
\text { d'activité }\end{array}$} & Banque et assurance & 4 & $30,8 \%$ \\
\hline & Commerce et Distribution & 2 & $15,4 \%$ \\
\hline & Transport & 2 & $15,4 \%$ \\
\hline & Télécom \& Service & 2 & $15,4 \%$ \\
\hline & Agroalimentaire & 2 & $15,4 \%$ \\
\hline & Pharmaceutique & 1 & $7,7 \%$ \\
\hline \multirow{2}{*}{ Sexe } & Masculin & 8 & $61,5 \%$ \\
\hline & Féminin & 5 & $38,5 \%$ \\
\hline \multirow{2}{*}{ Expérience } & $5-10$ ans & 4 & $30,8 \%$ \\
\hline & $10-15$ ans & 9 & $69,2 \%$ \\
\hline \multirow{8}{*}{$\begin{array}{c}\text { Poste } \\
\text { occupé }\end{array}$} & Directeur SI & 4 & $30,8 \%$ \\
\hline & Chef Département Support et SI & 2 & $15,4 \%$ \\
\hline & Responsable SI \& sécurité & 1 & $7,7 \%$ \\
\hline & Chef de projet SI & 2 & $15,4 \%$ \\
\hline & Responsable Administratif et Financier & 1 & $7,7 \%$ \\
\hline & Responsable Contrôle de Gestion & 1 & $7,7 \%$ \\
\hline & Responsable Ressources et Logistique & 1 & $7,7 \%$ \\
\hline & Chef de Division & 1 & $7,7 \%$ \\
\hline \multirow{2}{*}{$\begin{array}{c}\text { Type de } \\
\text { direction }\end{array}$} & SI & 9 & $69,2 \%$ \\
\hline & Métier & 4 & $30,8 \%$ \\
\hline
\end{tabular}

Tableau 3. Caractéristiques des interlocuteurs

Nous remarquons dans notre échantillon plus que la moitié sont des hommes et prés de $70 \%$ ayant une expérience supérieur à 10 ans. A noté que la totalité des répondants exerçant dans des postes de résponsabilité ce qui permet de bien cadrer notre étude puisque notre objectif est de tester le degré de conscience des responsables marocaines par rapport au concept d'alignement des SI en tant que déterminant crucial pour réaliser de résultats performants ainsi ces résponsables appartennant à des secteurs différents ce qui va permettre de donner une vision globale de ce concept dans notre terrain de recherche notamment dans l'étape confirmatoire.

L'analyse du contenu des entretiens semi-directifs menés lors de la phase exploratoire nous a permis de confirmer nos construits théoriques ainsi que l'identification de certains facteurs pertinents durant les différents entretiens et qui sont déjà développés dans la littérature traitant les SI. Nous rapportons dans ce qui suit, les résultats trouvés:

\section{Pertinence de la thématique dans le contexte Marocain}

Dans le but de tester le niveau de connaissance des interviewés et leur appréciation par rapport à notre objet de recherche on a décidé de commencer dans un premier temps par l'examen de leurs avis sur la question 
centrale de notre étude à savoir dans quelle mesure l'alignement des SI influence sur la performance? A ce propos la majorité des répondants ont confirmé l'importance et le rôle primordial des SI en tant que déterminant pour l'efficacité et la proactivité des organisations face aux changements de l'environnement, dans ce sens le concept d'alignement est présent implicitement dans ce processus de transformation de l'organisation. En outre la plupart des responsables sont conscients du poids ainsi que des sommes investis dans les SI et sont soucieux à ce que cet investissement contribue positivement à la performance de l'entreprise.

Extraits de verbatim qui illustrent notre propos:

"[...] Oui (il insiste 3 fois) aujourd'hui on assiste à une réelle révolution des SI sous l'égide de l'économie numérique ....faut le dire actuellement les systèmes d'information influencent toutes les activités et les entités des organisations (E1)».

" [...] Absolument (il a répondu avant même de terminer ma question). En effet la direction des SI commence à changer de visage (souriant) elle est passée d'une vision traditionnelle basée sur l'informatique à une autre plus stratégique ce qui implique fortement cette tendance actuelle des entreprises notamment en matière d'allocation des budgets important en SI (E3)».

"[...] L'augmentation du budget alloué aux SI est devenue une réalité incontournable ... ça c'est sûr toutefois ces SI peuvent contribuer à la performance de l'entreprise si ses dépenses sont bien maîtrisées et que les investissements dûment préparés en harmonie avec toutes les composantes de l'entreprise (E8)».

"[...] Dans nos jour on assiste à une nouvelle perception des SI dans la mesure où ils sont considérés comme levier de performance et une préoccupation majeur pour nous les praticien (E7)》.

" [...] Une chose très importante faut la prendre en considération, actuellement on parle de la bonne gouvernance des SI....non seulement on parle de l'importance de la contribution des SI aux entreprises, le mécanisme de l'alignement et l'un des piliers pour la réalisation de cette bonne gouvernance des SI (E9)».

" [...] Les SI aujourd'hui sont considérés parmi les déterminants de développement et de croissance de l'entreprise sur lesquels faut donner plus d'importance pour pouvoir faire face à une concurrence très rude dans ce contexte qui est en plein mutation (E2)».

Nous remarquons que les interlocuteurs insistent sur la valeur stratégique des SI et la place centrale occupée par ces derniers dans l'entreprise ainsi que sur leur contribution à l'amélioration de la performance et le développement et la croissance des entreprises, ce qui rejoint parfaitement notre revue de littérature portant sur cette question de la relation entre les SI et performance dont la majorité des travaux de recherches ont 
confirmé ce lien positif. Par conséquent, il convient de préciser que la présence seule des systèmes de l'information, même si ils sont très avancés, n'est pas une condition seule et essentielle pour garantir une bonne performance de l'entreprise. Surtout c'est grâce à leur articulation et adéquation avec toutes les composantes de l'entreprise notamment la stratégie, et l'infrastructure organisationnelle et technologique qu'elles peuvent améliorer la performance (Chan et al., 1997). Cela revient à dire que, l'alignement des SI résulte de la cohérence conjointe entre plusieurs choix notamment la stratégie SI et stratégie d'entreprise (alignement stratégique), la stratégie SI et la structure de l'organisation (alignement organisationnel), et enfin la stratégie SI et l'utilisation le choix des technologies (alignement technologique) (Rival, Y., 2005).

\section{- Axe n`1 : Alignement entre la stratégie d'entreprise et la stratégie SI}

Dans ce premier axe nous avons mis l'accent sur l'étude de la relation entre les deux stratégies d'entreprise et SI à travers le lien entre les directions des SI et métiers en termes d'élaboration de stratégies, la cohérence des objectifs fixés, les méthodes de planification collaboratives et le degré d'implication des parties prenantes et tous les facteurs potentiels qui peuvent influencer sur cette relation d'alignement.

L'analyse des entretiens révèle de nouveaux descripteurs dans ce processus de cohérence entre ces deux stratégies:

"[...]Actuellement les priorités stratégiques de notre entreprises consistent à améliorer la rentabilité économique, augmenter le chiffre d'affaire, accroitre davantage le portefeuille d'activités, développer de nouveaux produits/marchés, et la concrétisation de ces priorités passe impérativement en collaboration et des discussions régulières avec la direction SI pour tester la faisabilité technique de chaque objectif afin de mettre en place les solutions les plus adéquates (E12)».

"[...]...L'implication réciproque des deux directions en matière d'élaboration des stratégies et fixation des objectifs un parmi les facteurs clés de succès pour tous projets d'entreprise (E5)».

"[...]Pour le cas de notre entreprise il y a un comité de pilotage et de suivi présidé par le directeur général avec tous les responsables pour discuter et échanger tous les flux d'informations dont dispose chacun (E10)».

"[...] L'implication de la direction métier est très forte dans le processus d'alignement. Concernant la Direction SI, son rôle notamment la participation et la compréhension pour permettre la réalisation technique des besoins d'affaires exprimés et pour vous dire une chose dans notre société on accorde plus d'importance à la direction SI (E8)».

Par ailleurs, d'autres critères sont soulevés pour mieux réussir ce processus d'alignement comme le critère de la qualité de l'interaction des 
directions SI et métiers et ce à travers notamment la communication et la compréhension. Dans ce sens, E6 avance:

"[...] J'insiste sur la communication et l'échange régulier d'information entre les responsables lors de l'élaboration et de la mise en ouvre des stratégies ca va permettre une meilleure prise de décision... (E1)».

"[...] Dans notre entreprise des réunions hebdomadaire se font entre les responsables afin d'exposer l'état d'avancement et les problèmes rencontrés dans le but de faire aboutir le projet et de réussir les objectifs fixés par la direction générale (E2)».

"[...]...De ma part j'aimerai bien citer un élément d'une importance capitale dans la réussite de ce processus d'alignement à savoir la compréhension mutuelle et le degré d'engagement des parties prenantes (E4)».

Dans ce sens plus les DSI et les managers ont un langage simple et un partage mutuelle des connaissances, plus la compréhension mutuelle est importante et le niveau d'alignement sera élevé précisent (Preston \& Karahanna, 2009). C'est ainsi que les travaux de (Avison et al., 2004; Kerans \& Lederer, 2000 et 2003) montrent de leur côté la pertinence de l'alignement humain et de la communication entre les dimensions business et SI d'une entreprise.

(Papp \& Luftman, 1995) font remarquer, d'après une étude sur plus de 300 entreprises, qu'une faible communication entre les managers SI et les dirigeants d'affaires entrave la capacité d'alignement de l'organisation et constitue souvent un facteur déterminant d'échec des projets, d'où l'importance des réunions entre les responsables comme étant cité durant les entretiens.

"[...] Parfois les valeurs culturelles et comportementales du responsables SI influencent sur notre raisonnement et notre orientation dans la prise de décision... (Silence quelque seconde) certainement ceci est orientée dans l'intérêt de l'entreprise (E7)》.

"[...] Pour le cas de notre entreprise..., je constate que la culture propre et spécifique de chaque responsable est potentiellement a un effet sur l'orientation et la vision des responsables en matière d'élaboration des stratégies ce qui implique le poids qui peut avoir dans cette relation alignement et performance (E8)».

Pour réussir ce processus d'alignement et afin d'atteindre les objectifs fixés pour cela (E4) met l'accent sur la participation et l'engagement solidaire des parties prenantes internes à la définition et aux processus d'élaboration des orientations stratégiques.

[...] De ma part et à travers ma longue expérience dans le domaine des SI je considère que la compréhension des plans et des objectifs fixés par une organisation soient simple si le langage pratiqué est claire pour les 
collaborateurs ce qui va permettre d'avoir un alignement solide et durable avec un effet significatif sur la performance (E5)».

En effet, la quasi totalité des personnes interrogées ont accordé une attention particulière au rôle qui peuvent jouer par les parties prenantes dans l'amélioration $\mathrm{du}$ processus d'alignement des SI à la performance notamment les compétences, les valeurs culturelles des responsables en matière de l'élaboration des stratégies, le dialogue et l'échange des idées et des informations entre les managers ainsi qu'une meilleure compréhension des plans et des objectifs, l'ensemble de ces facteurs ne peuvent que influencer positivement sur cette relation alignement et performance qui reste aussi tributaire du degré d'implication des collaborateurs.

Dans la même lignée d'idée, ce qui a été avancé par les responsables interviewés a été déjà confirmé dans la littérature traitant cette question de la contribution des SI à la performance. Ainsi cela s'inscrit dans le sillage des travaux développés antérieurement notamment le cas de (Huang \& $\mathrm{Hu}$, 2007) qui ont accordé une importance capitale à la culture du Directeur des Systèmes d'Information (DSI) et à son effet modérateur sur l'alignement stratégique des SI. Ils insistent non seulement sur cette dichotomie mais encore sur un bon alignement entre la culture du manager et celle du responsable SI pour échapper d'une situation qualifiée de " culture gap ». Dans ce sens (Fimbel, 2007) reconnaît que la démarche de l'alignement stratégique dépend essentiellement des attitudes et des comportements des parties prenantes managériales et techniques impliquées dans ce processus et de leur interaction.

\section{Axe $n^{\circ} 2$ : Alignement entre l'infrastructure organisationnelle et la stratégie SI}

L'alignement entre la stratégie SI et l'infrastructure organisationnelle demeure déterminant dans la réussite des systèmes d'information. Ainsi, à noter que les SI ont devenu des catalyseurs de changement des organisations (Jouirou \& Kalika, 2004). A cet effet la structure organisationnelle est observée en tant que élément primordial en matière de choix stratégiques et technologiques pour une entreprise. Elle permet la déconcentration du pilotage et la délégation du pouvoir en facilitant la transmission et le partage de l'information au sein de l'entreprise (Kalika et al., 2002).

En effet, selon la majorité des chercheurs ainsi que des praticiens la performance d'une entreprise est tributaire du degré d'harmonisation entre sa structure et son environnement et ce dans le but d'atteindre une structure organisationnelle flexible par rapport aux changements de l'environnement. C'est ainsi que les SI jouent un rôle essentiel sur le plan de l'intégration jugée nécessaire pour garder une bonne cohérence au niveau du 
fonctionnement au sein de l'entreprise notamment la prise en compte du caractère transversal des relations entre les différents services.

Extraits de verbatim qui illustrent notre propos :

"[...] ...Au sein de notre entreprise on a une plateforme dédiée à l'échange et la coordination entre l'ensemble des collaborateurs .... Ce qui a permis de rendre l'information accessible et disponible à tous les niveaux hiérarchiques en la transmettant, en temps réel, en conséquence une forte réactivité enregistrée au niveau de notre entreprise (E5)».

"[...]Les collaborateurs communiquent par des courrier électronique avec leurs dirigeants en posant des questions relatives auxobjectifs à atteindre, les mesures à prendre, ainsi que aux nouvelles procédures organisationnelles, etc. Cette implication des collaborateurs est renforcée par des réunions hebdomadaires au cours desquelles les actions en cours sont présentées et discutées (E6)».

Cela qui implique que la mise en place d'un SI a permet, une amélioration au niveau du partage de l'information et une bonne communication entre les différents service qui était rendu plus facile et plus pratique qu'avant, dans ce sens « $\mathrm{E} 4$ » nous a donné un exemple relatif à une solution qui a été développé récemment suite à la constatation d'une perte de beaucoup de temps, une mauvaise gestion et coordination pour le service de comptabilité ainsi que des réclamations formulées à la direction générale. "[...] dans le cas d'une mission le collaborateur prend en charge tous le processus de déplacement par ses propres moyens à charge pour lui de présenter toutes les pièces justificatives et attendre environs 5 semaines pour le remboursement....maintenant avec l'intégration d'un nouveau module dans le SI on a pu rendre le délai de remboursement au maximum 10 jours et le collaborateur peut prendre des photos par téléphone depuis son hôtel ainsi le ticket du restaurant....et les envoyés en temps réel au service concerné pour prise en charge et préparation de règlement sans oublier la souplesse de gestion notamment moins de paperasse... tout ça dans le cadre de la création de nouveaux processus administratifs dédiés à l'activité au sein de l'organisation».

"[...] Toute information que soit stratégique ou opérationnelle est communiquée en temps réel sous format électronique à l'ensemble des collaborateurs au sein de l'entreprises (E12)».

"[...] Dans notre entreprise, on a une plateforme destinée au partage et la circulation des informations sous forme d'un compte rendu issu des réunions comprend l'ensemble des directives et des décisions prises par les responsables concernés (E10)».

De manière générale la majorité des interlocuteurs ont insisté sur la nécessité de la cohérence entre l'infrastructure et le processus organisationnel avec la stratégie SI pour avoir un meilleur partage de 
l'information et la coordination entre les différentes entités de l'entreprise et particulièrement de faciliter la prise de décision ainsi que les orientations stratégiques des directions. Dans ce sens, (Tavakolian, 1989; Currie, 1996) ont noté que les SI permettaient, au sein d'une entreprise décentralisée, d'attribuer l'autorité et les décisions en rendant plus fluide la diffusion mais aussi le partage d'information dans l'ensemble de l'organisation. Aussi d'autres chercheurs comme (Raymond, Paré \& Bergeron, 1995) ont trouvé que la relation entre la sophistication des SI et la sophistication de l'infrastructure organisationnelle était significativement plus intensive dans des PME à haute performance que dans celles à basse performance.

\section{Axe n`3 : Alignement entre l'infrastructure technologique et la stratégie SI}

Nous avons traité avec les répondants les questions relatives à la gestion de l'infrastructure technologique dans la mesure où cette infrastructure constitue le socle de toutes les activités réalisées par l'entreprise. Il s'agit notamment d'étudier comment l'entreprise choisit et utilise de nouvelles applications SI pour appuyer le développement de ses activités, à ce niveau il convient de souligner que (Bergeron et al., 2001; Croteau et al., 2001) ont constaté que l'ajustement dans l'utilisation des technologies constitue un facteur clé pour atteindre un degré de performance satisfaisant Elle traduit la cohérence entre la stratégie TI et l'infrastructure technologique liée aux TI (Broadbent \& Weill, 1993; Henderson \& Venkatraman, 1993).

Extraits de verbatim qui illustrent notre propos :

«[...] ....Dans notre établissement une gestion très rigoureuse accordée en matière de contrôle des ressources technologique ...matériels et logiciel cela veut dire qu'on veille à une meilleur gestion de l'infrastructure et on est conscient du rôle central de ces ressources dans le bon fonctionnement de l'entreprise...(E1)».

"[...] ...je tiens à vous préciser que la gestion de l'infrastructure est l'axe le plus technique de la DSI, et la moindre fausse note est remarquée et accompagnée d'un 'C'est encore et toujours la faute de l'informatique !' ...ceci pour vous dire des ateliers de cadrage sous forme de réunion se font conjointement avec les autres entités de l'entreprise notamment dans le cadre des projets d'investissements en SI (E9)».

"[...] ....investissement en SI ... (silence quelque seconde) c'est le processus d'urbanisation en question ... on est souvent impliqué et régulièrement à ce type de décision qui touche notre parc informatique notamment sur les critères de sélection (E13)».

"[...] ...oui on assiste à des ateliers formations qui portent sur les dernières tendances en matière de développement des SI et cela s'inscrit dans le 
sillage de la politique de veille technologique adoptée par notre groupe (E2)».

\section{Axe nº 4 : Performance de l'entreprise}

Dans l'objectif de savoir la réaction des responsables des entreprises objet de l'étude exploratoire quant au l'effet des SI sur la performance dans le contexte marocain, A ce niveau il est a noté que la majorité de recherches traitant la question portant sur la relation alignement/performance mettent en avant l'effet positif de l'alignement sur la performance, quels que soient la nature et le nombre de facteurs étudiés. L'alignement de la fonction SI avec les autres composantes de l'entreprise favorise ainsi sa performance (Karoui, 2012; Rival, 2005; Jouirou \& Kalika, 2004; Bergeron, Raymond, \& Rivard, 2002; Sabherwal \& Chan, 2001; Bergeron \& Raymond, 1995; Bergeron et al., 1998; Croteau et al., 2001; Chan et al., 1997; Teo \& King, 1996; etc.). Dans ce sens l'apparition de la notion d'alignement qui semble être un impératif voire une condition sine qua non pour les entreprises soucieuses de leur performance.

L'analyse des entretiens révèle le caractère multidimensionnel du construit de la performance dans l'esprit des responsables interviewés, le premier critère généralement d'ordre financier c'est le cas de résultats financiers, comme a été souligné clairement dans les propos suivants :

"[...] Etre performant, c'est tout d'abord avoir un bilan positif en fin d'année (E6)».

"[...] ...maitrise des coûts par exemple récemment grâce à la mise en place d'une petite solution SI dans la chaine de boucherie de notre groupe on a pu augmenter notre $C A$ et diminuer les charges afférente notamment à la passation de commande (souriant) sans cité le pourcentage (E8) ».

"[...] ... Notre portefeuille des clients a été doublé sur un laps du temps très court cela dû à l'implémentation d'un SI dédié à la gestion des clients qui a été très bénéfique pour notre entreprise notamment sa réputation sur le marché (E5)».

"[...]...Oui le retour sur investissement est satisfaisantl'enveloppe financière destinée à cette fin a été récupéré de même avec une plus-value (E12) ».

"[...] Pour notre cas, oui on peut parler de la réalisation de bonne performance...une amélioration de la productivité a été constaté suite à la mise en cuvre d'un SI au sein de notre entreprise (E3) ».

"[...]...Une amélioration au niveau de l'efficacité et la flexibilité a été enregistré pour notre groupe par rapport aux concurrents cela grâce aux efforts déployés par la direction générale et la direction des SI en matière d'investissement en SI ce qui a permet de consolider notre croissance dans un environnement très concurrentiel (E7)». 
Il convient de remarquer que les réponses des répondants montrent que chaque entreprise donne une priorité à une dimension de performance principalement financière (résultat financier positif, baisse des coûts...) et commerciale (augmentation du CA, la part du marché...), et la plupart confirment l'apport des SI à la performance en tant que levier stratégique dans le contexte des entreprises étudiées ce qui rejoint les travaux antérieures portant sur cette thématique.

\section{Modèle conceptuel et hypothèses de recherche}

Dans le cadre de cette étude qualitative, l'élaboration de notre modèle conceptuel a été effectuée sur la base du modèle initial d'alignement stratégique de (Henderson \& Venkatraman, 1993), et sur d'autres travaux de recherches traitant cette question de la performance des entreprises induite par les SI (Karoui, 2012; Rival, 2005; Jouirou \& Kalika, 2004) en plus l'intégration d'un ensemble de variables d'extension issues des travaux de recherches de (Schlosser et al., 2012; Mezghani \& Mezghani, 2008) qui ont été confirmés suite à la réalisation des entretiens semi-directifs auprès des responsables métiers et SI de treize entreprises marocaines à savoir l'interaction des parties prenantes en terme de compétence, culture, communication, compréhension et la méthodologie et plan pour l'élaboration des stratégies. S'ajoute à cela les recommandations formulées par (Reix \& Rowe, 2002) qui stipulent que le modèle d'alignement stratégique porte une approche technique et managériale des SI qui tend à négliger notamment l'apport humain.

Notre modèle fait recours à un ensemble de variables issues de notre revue de la littérature ainsi que les résultats de l'étude exploratoire qualitative ont permis de mettre en exergue en définitif de trois blocs de variable du modèle à tester:

- $\quad$ Variable dépendante : Performance d'entreprise.

- Variables explicatives: Stratégie SI, stratégie d'entreprise, infrastructure organisationnelle et infrastructure technologique.

- Variables modératrices : Dimensions sociales, dimensions humaines, dimensions intellectuelles. 


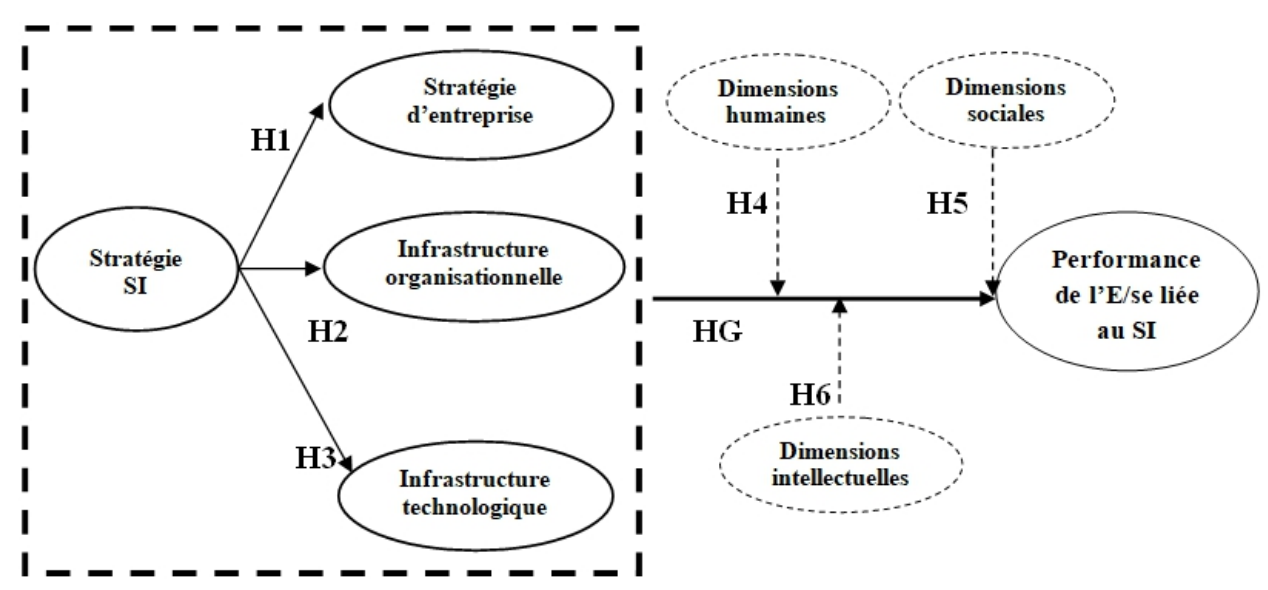

Figure 3. Modèle de recherche

Ainsi, nous avons formulé 6 hypothèses relatives à notre modèle conceptuel de recherche:

HG: L'alignement de la stratégie SI, de la stratégie d'entreprise, de l'infrastructure organisationnelle, et de l'infrastructure technologique influence positivement la performance de l'entreprise.

H1: L'alignement entre la stratégie SI et la stratégie d'entreprise influence positivement la performance de l'entreprise.

H2: L'alignement entre la stratégie SI et l'infrastructure organisationnelle influence positivement la performance de l'entreprise.

H3: L'alignement entre la stratégie SI et l'infrastructure technologique influence positivement la performance de l'entreprise.

H4: La dimension humaine a une influence sur la relation alignement des SI et la performance de l'entreprise

H5: La dimension sociale a une influence sur la relation alignement des SI et la performance de l'entreprise.

H6: La dimension intelectuelle a une influence sur la relation alignement des SI et la performance de l'entreprise.

\section{Conclusion}

Notre étude s'inscrit dans le cadre dans une approche qualitative à caractère exploratoire hybride, ainsi la méthodologie adoptée repose sur les entretiens semi-directifs, répétés jusqu'à ce que la saturation théorique soit atteinte. Nous avons veillé à maximiser la diversité des profils des participants afin d'accroître le potentiel de découverte de notre recherche. Toutefois, nous avons fait le choix des éléments qui sont basés sur le jugement du chercheur dans le cadre d'un échantillon de choix raisonné. Au total, l'échantillon final est constitué de treize personnes. 
En effet l'apport de cette étude exploratoire consiste à l'élaboration de modèle hypothétique de recherche. Partant d'une approche d'évaluation multidimensionnelle via une démarche sociotechnique de l'alignement stratégique et un cadre théorique basé sur deux théorie, la contingence en tant que théorie principale et la théorie des parties prenantes en tant que complémentaire, nous avons pu proposer notre modèle final qui décrivent l'ensemble des relations contribuant à la formation de la performance induite par la mise en place des SI. En se basant sur les deux premières étapes du paradigme de Churchill (spécification du domaine du construit et la génération d'un échantillon d'items) pour entamer les étapes suivantes afin de tester le modèle conceptuel proposé, via une approche quantitative confirmatoire pour permettre la validation ou le rejet de nos hypothéses de recherche par le recours aux méthodes des équations structurelles qualifiées de méthodes d'analyse multivariée de la deuxième.

Sur le plan théorique ce travail de recherche a 1'ambition de proposer un modèle théorique au croisement de deux perspectives théoriques de manière simultanée à savoir la théorie de la contingence et la théorie des parties prenantes à travers la mobilisation de deux approches d'évaluation de la contribution des SI à la performance une causale (alignement stratégique) et autre processuelle ( sociotechnique).

Sur le plan manageriel cette étude a permis aux responsables interviwés d'être conscients et vigilants quant à l'impératif de l'alignement des SI comme déterminant d'une bonne performance. dans ce sens en se basant sur les travaux de (Fimbel ,2006) et de (Sauer \& al 1997) stipulent qu'une faible performance de l'entreprise est due un déficit de leur alignement avec toutes les composantes de l'entreprise notamment l'absence de cohérence entre la stratégie de l'entreprise et celle des SI ce qui peut entraîner une certaine confusion par rapport à la prise de bonnes décisions adéquates pour atteindre les objectifs de performance tracés. Autrement dit pour améliorer la performance de leurs firmes et tirer profit de leurs investissements en SI, il est indispensable pour les dirigeants de s'assurer de la cohérence entre la stratégie d'affaires et la stratégie SI, infrastructures organisationnelle et technlogique.

\section{References:}

1. Albarello, L. (2004). "Apprendre à chercher, l'acteur social et la recherche scientifique“, Bruxelles : De Boeck Université., page 65.

2. Allouani, S.A. (2013). "Les déterminants relationnels de la performance export contribution a travers le concept de capacités relationnelles - Cas des PME au Maroc" thése de doctorat en sciences de gestion à l'université Hassan II Settat. 
3. Avison, D., Jones, J., Powell, P. \& Wilson, D. (2004). "Using and Validating the Strategic Alignment Model", Strategic Information Systems, Vol. 13, No. 3, pp. 223-246 Bardin, L. 2003. "L'analyse de contenu“", Paris, PUF, $289 \mathrm{p}$

4. Bennani, A., Beldi, A. \& Baile, S. (2004). "Dix ans de recherche en alignement : 1993-2003“. In $9^{\text {ème }}$ Congrès de l'AIM, Evry (INT).

5. Bergeron, F. \& Raymond, L. (1995). "The contribution of IT to the Bottom Line: A Contingency Perspective of Strategic Dimensions", ICIS Conference, 167-181.

6. Bergeron F., Raymond L. \& Rivard, S. (2001). "Fit in Strategic Information Technology Management Research: An Empirical Comparison of Perspectives", Omega, 29 (2), pp. 125-142.

7. Bergeron, F., Raymond, L. \& Rivard, S. (2002). "Strategic Alignment and Business Performance: Operationalizing and Testing a Covariation Model", working paper HEC Montréal, May.

8. Bergeron, F., Raymond, L. \& Rivard, S. (2004a). "Ideal Patterns of Strategic Alignment and Business Performance", Information \& Management, Vol. 41, No. 8, pp. 1003-1020.

9. Bergeron, F., Raymond, L. \& Rivard, S. (2004b). "L'alignement stratégique des TI et la performance des PME ", 13ème Conférence de l'AIMS, Normandie, Vallée de la Seine, 2, 3 et 4 Juin.

10. Bharadwaj, A. (2000). "A resource-based perspective on information technology capability and firm performance: an empirical investigation. » Mis Quarterly: 169-196

11. Blanchet, A. \& Gotman, A. (1992). "L'enquête et ses méthodes : l'entretien", Nathan, Paris

12. Broadbent, M. \& Weill, P. (1993). "Improving business and information strategy alignment: Learning from the banking industry, "IBM Systems Journal, 32 (1), pp. 162-177.

13. Brownc, V. \& Magill, S.L. (1994). "Alignment of the IS Functions with the Enterprise: Toward a Model of Antecedents ", Management Information Systems Quarterly, December, p. 371-403.

14. Cigref (2003). "Les Parties Prenantes du Système d'Information : un nouveau regard sur la maîtrise d'œuvre et la maîtrise d'ouvrage. ", www.cigref.

15. Chan, E. Y. \& Reich, H. B. (2007). "IT alignment: what have we learned? (State of the A rt) ". Journal of Information Technology, $\mathrm{N}^{\circ} 22, \mathrm{p} .297-315$

16. Chan, Y.E., Huff, S.L., Barclay, D.W. \& Copeland, D.G. (1997). "Business Strategic Orientation, Information Systems Strategic 
Orientation, and Strategic Alignment", Information Systems Research, 8, 2, 125-150.

17. Chan, Y.E. \& Huff, S.L. (1993). "Strategic information systems alignment", Business quarterly, fall, 58, 1, 51-56.

18. Chandler, A.D. (1962). "Strategy and Structure: Chapters in the History of the American Industrial Enterprise ", MIT Press, Cambridge, Massachusetts.

19. Charreire, S. \& Durieux, F. (1999). "Explorer et tester ", in Méthodologie de Recherche en Management, coordonné par Raymond-Alain Thiétart, Dunod, chapitre 3, pp.57-80

20. Chedia K, (2012). “Alignement Des Technologies De L'information: Déterminant De La Performance (Cas des Entreprises Tunisiennes) Global Journal of Management and Business Research Volume 12 Issue 5 Version 1.0 March.

21. Churchill, G. A., Jr. (1979). A paradigm for developing better measures of marketing constructs. JMR, Journal of Marketing Research, 16(1), 64-73

22. Croteau, A.M. \& Bergeron F. (2001). "An Information Technology Trilogy: Business strategy, technological deployment and organizational performance", Journal of Strategic Information Systems, 10(2), pp. 77-99.

23. Croteau, A.M., Bergeron, F. \& Raymond, L. (2001). "Comportement stratégique, choix et gestion des systèmes d'information : contribution a la performance ", Systèmes d'Information et Management, 4(6).

24. Currie, W L. (1996). "Organizational structure and the use of IT: Preliminary findings of a survey in the private and public sector", International Journal of Information Management, 16 (1), pp. 51-64

25. De Vaujany F-X. (2009). Les grandes approches théoriques du système d'information. Lavoisier.

26. Evrard,Y., Pras, B \& Roux, E. (2003). "Market : études et recherches en marketing “, Dunod, $3^{\text {ème }}$ édition

27. Fimbel, E. (2007). "Alignement Stratégique : synchroniser les SI avec les trajectoires et manœuvres des entreprises" .Paris, Ed. Pearson, collection Village Mondial, Aout, p.360.

28. Freeman, R. E. (1999). "Divergent Stakeholder Theory. “ Academy of Management Review, $\mathrm{Vol}^{\circ} 24, \mathrm{~N}^{\circ} 2$, p.233-236

29. Gervais, M. (2001). Contrôle de Gestion, Economica, Paris.

30. Henderson, J.C. \& Venkatraman, N. (1993). "Strategic Alignment: Leveraging Information Technology for Transforming Organizations", IBM Systems Journal, 32, 1, 4-16. 
31. Huang, C.D. \& Hu Q. (2007). "Achieving IT-Business strategic alignment via enterprise-wide implementation of balanced scorecards", Information Systems Management, Vol 24, pp173-184.

32. Jouirou, N. \& Kalika, M. (2004). "Strategic alignment: a performance tool (an empirical study of SMEs)", AMCIS Conference, New York.

33. Iivari, J. (1992). "The organizational fit of information systems ", Journal of information systems, 2, 1, 3 -29

34. Kalika, M., Bellier, S., Leroy, I., Isaac, H., \& Josserand, E. (2002). "Le e-management, vers l'entreprise virtuelle ? L'impact des TIC sur l'organisation." Editions Liaisons, Paris.

35. Kearns, G. S. \& Lederer, A. L. (2000). "The effect of strategic alignment on the use of IS based resources for competitive advantage", The Journal of Strategic Information Systems, Vol. 9, No. 4, pp. 265-293.

36. Kearns, G. S. \& Lederer, A. L. (2003). “A Resource-Based View of Strategic IT Alignment: How Knowledge Sharing Creates Competitive Advantage", Decision Sciences, Vol. 34, No. 1, pp. 129.

37. Kefi, H. \& Kalika, M. (2003). "Choix stratégiques de l'entreprise étendue et déploiement technologique : alignement et performance ", Conférence AIM, $22 \mathrm{p}$.

38. Leidner, D. E. \& Kayworth, T. (2006). "A Review Of Culture In Information Systems Research: Toward A Theory Of Information Technology Culture Conflict", MIS Quarterly, Vol 30, N. 2, pp 357399.

39. Luftman, J. (2000). “Assessing business-IT alignment maturity", Communication of the AIS, 4, 14, 1-50.

40. Mezghani, K. \& Mezghani, L. (2008). "Rôles des compétences managériales des responsables des affaires dans l'alignement stratégique des ERP : proposition d'un modèle de recherche ", Actes du 17ème colloque de l'AIMS, Nice, France, 28-31 mai

41. Mercier, S. (2005). Théorie des Parties Prenantes et Management stratégique : revue, synthèse et perspectives de la littérature en gestion. "In 14ème Conférence de l'Association Internationale de Management Stratégique AIMS, Angers, les 6, 7, 8 et 9 Juin 2005

42. Muhanna, W. A. \& Stoel, M. D. (2010). "How do investors value IT? An empirical investigation of the value relevance of IT capability and IT spending across industries. Journal of Information Systems, 24(1), 43-66.

43. Papp, R. \& Luftman J. (1995). "Business and IT Strategic Alignment: New Perspectives and Assessments ", AMCIS, 5 p. 
44. Preston, D. S. \& Karahanna, E. (2009a). "How to develop a shared vision: The key to IS strategic alignment“. MIS Quarterly Executive, 8 (1), 1-8.

45. Preston, D. S. \& Karahanna, E. (2009b). "Antecedents of IS strategic alignment: A nomological network. "Information Systems Research, 20 (2), 159-179.

46. Raymond, L., Croteau, A.M. \& Bergeron F. (2010). "La performance des TI expliquée par leur rôle stratégique, leur gestion et leur utilisation ", Le $15^{\text {ème }}$ colloque de 1'AIM, La Rochelle (France).

47. Raymond, L. (2002). "L'impact des systèmes d'information sur la performance de l'entreprise. In ROWE, F. Faire de la recherche en Systèmes d'Information“. Paris, Ed. Vuibert, FNEGE, Septembre, p.355

48. Raymond, L., Paré, G. \& Bergeron, F. (1995). "Matching Information Technology and Organization Structure: An Empirical Study with Implications for Performance". European Journal of Information Systems, 10 (4) 3- 16.

49. Reix, R. \& Rowe, F. (2002). "La recherche en Systèmes d'Information: de l'histoire au concept. In ROWE, F. Faire de la recherche en Systèmes d'Information. Paris, Ed. Vuibert, Fnege, Septembre.

50. Reich, B. H. \& Benbasat (1996). "I. Measuring the linkage between business and information technology objectives". MIS Quarterly, , $\mathrm{Vol}^{\circ} 20, \mathrm{~N}^{\circ} 1, \mathrm{p} .55$

51. Renaud, A. (2012). "Traduire pour aligner : Une analyse par les pratiques de la conduite de projets de reconfigurations de systèmes d'information " Thèse de doctorat en sciences de gestion, université Paris Dauphine

52. Rival, Y. (2008). "Une exploration des mécanismes formateurs de la performance de l'entreprise liée à Internet", Conférence AIMS, Germany.

53. Rival, Y. (2005). “L'évaluation de l'activité Internet de l'entreprise : une application au secteur du tourisme français " Thèse de doctorat en sciences de gestion, université Paris Dauphine.

54. Roussel, P. \& Wacheux, F. (2006). "Management des ressources humaines : méthodes de recherche en sciences humaines et sociales“. Bruxelles: De Boeck.

55. Santhanam, R. \& E. Hartono (2003). "Issues in Linking Information Technology Capability to Firm Performance “ MIS QUARTERLY 27(1): 125- 154 
56. Sabherwal, R. \& Chan, Y. (2001). "Alignment Between Business and IS Strategies: A Study of Prospectors, Analyzers, and Defenders, Information Systems Research," 12(1), pp. 11- 33.

57. Saulquin, J.Y. \& Schier, G. (2005). "La RSE comme obligation/occasion de revisiter le concept de performance ? ", Actes du congrès La responsabilité sociale de l'entreprise : réalité, mythe ou mystification?

58. Schlosser, F., Wagner, H.-T. \& Coltman, T. (2012). "Reconsidering the Dimensions of Business-IT Alignment", 45th Hawaii International Conference on System Sciences.

59. Teo, T. \& King, W.R. (1996). "Assessing the impact of Integrating Business Planning and IS Planning », Information \& Management, Vol. 30, $\mathrm{N}^{\circ} 6$, p. 309-321.

60. Teo, T. S. H \& King, W. R. (1997). "Integration between business planning and information systems planning: validating a stage hypothesis“. Decision Sciences,28, Spring,279-308.

61. Tavakolian H. (1989). "Linking the Information Technology Structure with Organizational Competitive Strategy: A survey", MIS Quarterly, 13 (3), pp. 309-317.

62. Venkatraman, N. (1989a). "Strategic Orientation of Business Enterprises: The Construct, Dimensionally and Measurement". Management Science, 35 (8) 942- 962.

63. Venkatraman, N. (1989b). "The Concept of Fit in Strategy Research: Toward Verbal and Statistical Correspondence. " Academy of Management Review, 14 (3) 423- 444

64. Venkatraman, N. \& Ramanujam, V. (1986). "Measurement of Business Performance in Strategy Research: A Comparison of Approaches", The Academy of Management Review, Vol. 11, No. 4, pp. 801-814

65. Verreault, D. \& Rivard, S. (2003). "Alignement stratégique des technologies de l'information: une perspective basée sur les ressources " Chaire de gestion stratégique des technologies de l'information HEC Montréal

66. Wang, E T G, Shih, S-P, Jiang, J.J \& Klein, G. (2008). “The Consistency among Facilitating Factors and ERP Implementation Success: A Holistic View of Fit". Journal of Systems and Software, 81 (9), 1609-1621.

67. Weill, P. \& Olson, M.H. (1989). "An Assessment of the Contingency theory of management Information Systems", Journal of Management Information Systems, Vol. 6, $\mathrm{n}^{\circ} 1$, p. 59-86..

68. Y. Regragui (2016). "Impact of Information Systems on the Performance from an Alignment Approach: Conceptual Research 


\section{Model" The International Journal of Business \& Management Volume IV, Issue VIII, August.}

Annexe 1. Principaux thèmes du guide d'entretien destinés aux responsables métiers et SI

\begin{tabular}{|c|}
\hline Questions sur la pertinence de la thématique dans le contexte marocain. \\
\hline \begin{tabular}{c} 
Présentation de l'entreprise.( Activité , organisation et le SI utilisé...) \\
Evolution de la thématique dans les entreprises. \\
- Niveau de connaissance et le degré d'implication des responsables. \\
Questions sur la cohérence des choix stratégique \\
Les Priorités stratégiques ? \\
Implication des deux directions métier et SI dans l'élaboration des stratégies de l'entreprise ? \\
Communication et dialogue entre les parties prenantes ? \\
L'importance accordée aux SI dans l'entreprise ? \\
Le rôle de la compétence des responsables en matière de planification et de l'élaboration des \\
stratégies d'entreprise et SI. \\
\hline Questions sur la cohérence dans la conception de l'organisation \\
\hline Coordination, échange et partage de l'information ? \\
\hline Question sur la cohérence dans l'utilisation technologique \\
\hline Evolution technologique et complémentarité des investissements lies aux SI. \\
Veille technologique ? \\
\hline Questions sur la performance induite par les SI. \\
\hline L'apport des SI en termes de rentabilité, part du marché, innovation, baisse des coûts et \\
croissance de l'entreprise?
\end{tabular} \\
\hline
\end{tabular}

